Patricia May Ververs, Michael C. Dorneich, Michael D. Good, Joshua Lee Downs (2002). "Integrating critical information on flight deck displays," to appear in The Proceedings of the $46^{\text {th }}$ Annual Meeting of the Human Factors and Ergonomics Society, Baltimore, MD, September 30-October 4, 2002.

\title{
INTEGRATING CRITICAL INFORMATION ON FLIGHT DECK DISPLAYS
}

\author{
Patricia May Ververs, Michael C. Dorneich, Michael D. Good, Joshua Lee Downs \\ Honeywell Laboratories \\ 3660 Technology Drive \\ Minneapolis, Minnesota
}

\begin{abstract}
Honeywell Laboratories has developed a concept for integrating multiple sources of data concerning information outside the aircraft. The concept, ANCOA (Alerting and Notification for Conditions Outside the Aircraft), was conceived as means for reducing error conflicts and establishing a clear prioritization among currently independent and disparate alerting systems for hazards external to the aircraft (e.g., TCAS, EGPWS). This paper documents an empirical evaluation of ANCOA by 12 professional pilots. The concept was evaluated in Honeywell Laboratories' Flight Simulation Laboratory in Minneapolis, Minnesota. Two core ANCOA features were manipulated and compared. The first was the integration of information by comparing ANCOA's integrated, overlaid features to a traditional display layout where the information was available on separate displays. The second variable was the categorization for incoming alerts (traffic, terrain, weather, scheduling constraints) by comparing alerts sorted by category to those without a category differentiation. Data support the integration of currently disparate systems onto a single display with performance requiring fewer pilot inputs and yielding lower workload scores. Categorization had little influence on pilot performance.
\end{abstract}

\section{INTRODUCTION}

This paper presents an empirical evaluation of the Alerting and Notification of Conditions Outside the Aircraft (ANCOA) concept that was developed as a means to present alerting and notification information about conditions external to the aircraft. Current alerting systems warn of time-critical terrain (e.g., Enhanced Ground Proximity Warning System, EGPWS), traffic (e.g., Traffic Collision and Avoidance System, TCAS), or various weather phenomena (e.g., reactive windshear). Other independent alerting systems recently developed include turbulence detection systems, predictive windshear, etc. These newer alerting systems typically trigger their alerts via complex algorithms based on multiple parameters, many of which are not readily available to the pilot. Thus, it is no longer easy for pilots to interpret or verify alert credibility (Pritchett, Vandor, \& Edwards, 1999). This lack of information can lead to misguided trust in automation (Lee \& Moray, 1992), over- and under-reliance on automation (Riley, 1996; Parasuraman \& Riley, 1997), authority versus responsibility issues (Woods, 1986), and a host of humancomputer interaction issues. To add to the complexity, Automatic Dependent Surveillance - Broadcast (ADS-B), which will extend the range of traffic alerts and various electronic communication links, are beginning to allow much more information to reach the crew from ground sources. However, there is no standard protocol for prioritizing, organizing, and integrating the information to reduce the demands on pilot attention and information processing. Again, these alerting systems are separate, independent units manufactured by a variety of avionics suppliers, each using different alerting and display philosophies to present information to the crew. This lack of integration has lead to conflicting or contradictory information and resulted in documented incidents (See Ververs, Good, Rogers, Riley, \& Dorneich, 1999).
In response to the increasingly complex flight environment, Honeywell Laboratories developed a concept that addresses the emerging problems. This system is based on the application of human-centered guidelines and the development of a consistent philosophy for designing the components of an integrated aural and visual alerting system. ANCOA integrates information from independent alerting systems enabling multiple alerts of external conditions to be prioritized and de-conflicted before being presented to the flight crew. The goal was to support prompt and appropriate responses to adverse conditions based on good situation awareness. We have validated the resulting concept in an empirical evaluation with commercial pilots in a fixed-based, medium fidelity simulator.

\section{The ANCOA Concept}

Clear alerting and display philosophies were established with ANCOA to present information to the crew, thereby creating a standard protocol for prioritizing and organizing the information to reduce the demands on pilot attention and information processing. Specifically, the concept includes five basic components: (1) the ability to de-conflict currently separate alerts such as TCAS and EGPWS to avoid confusion on the appropriate action to take; (2) categorization (weather, traffic, ground, scheduling constraints) and prioritization (time-critical, tactical and strategic) of alerts to reduce pilot information processing requirements; (3) directional, multidimensional aural cueing to allow quick "pre-processing" of the condition (this aids in time-critical responses and in deciding the priority of the alerted condition relative to the ongoing task); (4) integrated graphic presentation of conditions external to the aircraft to support better situation awareness; and (5) ability to grow and expand to incorporate new technologies and added functionality. 


\section{Alerting Philosophy}

An effective alerting system needs to support six basic information processing (IP) functions: detection, localization, categorization, prioritization, description, and response. In ANCOA, the functional requirements are mapped to a continuum of aural and visual display components. Aural displays are best used for initial detection and localizing threats, while visual displays are effective for conveying detailed information via text and graphics. Though ANCOA supports each of the IP functions, for the purposes of this evaluation we focused on the categorization and prioritization schemes. Four unique categories were used to classify the conditions outside the aircraft: traffic, weather, terrain, and scheduling constraints. Each category was visually and aurally represented in the alert to the crew either through voice and text warnings announcing the threat or aural 'earcons' and visual icons denoting the condition. The prioritization scheme consisted of three levels primarily based on the time until impact on mission and certainty of the condition. In general, conditions were considered (1) time-critical if the crew needed to respond between 0-60 seconds, (2) tactical if the threat was between 1-10 minutes away or if the condition had a longer timeframe but was $100 \%$ certain (e.g., equipment outage at destination airport), or (3) strategic if the condition was more than 10 minutes away and less than $100 \%$ certain. The prioritization scheme was one method used for enabling the integration of multiple systems but still providing the flight crew with the necessary information to determine the highest priority threat. The ANCOA concept is an implementation of the above alerting philosophy and was evaluated in the experiment described below.

\section{METHODS}

The study gathered empirical data concerning two core ANCOA features: integration and categorization. We assessed whether visually integrating multiple sources of information will allow the pilots to easily identify interrelationships between multiple sets of data. We compared ANCOA to a traditional display layout where the information is available on separate displays. We expected pilots to detect and identify the adverse condition more quickly with ANCOA thereby providing more time for generating an appropriate response. We also expected the integrated presentation format to ensure greater situation awareness leading to more appropriate decisions. When response recommendations or directives were provided by ANCOA, we expected pilots to respond more quickly given they had a more complete account of the situation.

We also investigated the categorization of alerts into the four distinct classes (terrain, weather, traffic, scheduling constraints). We compared alerts sorted by category to those without a category differentiation. We provided categorical information embedded in the aural tone and in the visual message. We investigated whether this information would affect identification times. We expected the ANCOA categorization to enable the pilot to more quickly identify the source of the problem with little or no residual affect on secondary tasks.

\section{Experimental Design}

The experimental design was a 2 (integrated vs. separate) x 2 (category vs. no category) x 12 (scenarios) design. The integrated display condition enabled the pilot to overlay each of the functions (terrain, traffic, map, and weather). In the separate display condition each the functions operated as mutually exclusive modes, that is, when the pilot selected one mode the previously displayed mode was replaced. The alerts in the category condition had unique aural tones assigned with each category type (traffic, terrain, weather, scheduling constraints). Additionally, a small icon representing the category was displayed before each of the text messages. The no category condition had the same generic aural cue to represent all the alerts and no icon was associated with the message.

\section{Participants}

Twelve commercial pilots (nine Captains, two First Officers, and one Flight Engineer), ranging in age from 31 years to 56 years, with an average age of 45 years and a mean of 10,700 flight hours, were recruited for this evaluation. Half of the pilots had experience in glass cockpit aircraft.

\section{Procedure}

Upon arrival, pilots were given the instructions and consent and demographics forms. Once in the flight simulator, the experimenter reviewed the flight simulator controls and displays with the pilot, and each pilot was given the opportunity to practice flying the simulator and responding to sample terrain, traffic, and weather alerts in both the integrated and separate display conditions. The pilot sat in the left seat and had the role of pilot flying and the experimenter was the first officer. The experimenter instructed the pilot that his tasks were to fly to the specifications given to him at the beginning of each of the 12 scenarios and to respond to any alerts. Tasks included flying to target airspeed, heading, and altitudes. Their responses to the alerts were measured along with their response times. The pilots were instructed to first respond to the alert by announcing the category type. The experimenter recorded their response. The experimenter also manually recorded when they had resolved the situation. The pilots worked under high workload conditions given that they hand-flew an unfamiliar aircraft with manual throttles in a mountainous area, usually under poor visibility conditions. The only available information was the out-the-window view of the environment, a primary flight display (PFD) with heading, airspeed, altitude, radio altitude, attitude direction indicator (ADI), and the main ANCOA displays, the Message Alerting and External Situation Awareness (MAESA) display and the 3D aural display. At the conclusion of each scenario, pilots were given a short post-scenario questionnaire and the NASA-TLX workload rating scale (Hart \& Staveland, 1988). At the end of the experiment, subjects were given a postexperiment questionnaire and debriefed. The entire experiment was videotaped and lasted about 3.5 - 4 hours per subject. 


\section{Displays}

There were three main displays used in the implementation of the ANCOA concept: MAESA, a primary flight display (PFD), and a 3D audio display.

$M A E S A$. This display graphically represented the relevant external threats to the aircraft. See Figure 1 at end of paper. Function buttons located along the left side of the display enabled the pilot to display the terrain, weather, traffic, and map information. Depending on the integration condition this information could be overlaid (integrated) or served in a modal manner displaying only a single condition at a time (separate). Each of the primary functions (e.g., weather) had subfunctions (e.g., winds) for further modifying the information presentation. Care was taken to allow the external information to be overlaid while ensuring that no individual details would be obscured. Figure 1 indicates how weather (i.e., circular cells) could be overlaid over terrain (i.e., chevrons) without losing relevant feature information. Though shown in black and white in the figure, the display was colorcoded. Pilots navigated through the information space using the eight outside arrows that surrounded the graphical display area. Once an arrow was pressed the display entered an exocentric presentation mode as opposed to standard, aircraftcentric and the pilot could navigate ahead in the flight plan. There was also a dedicated message display area, located on the right of the display. Time-critical and tactical alerts were separated from the strategic messages. There was a log area indicating the message history listed by the time the message was received. A single line of text represented each message, however, more details were available in a dedicated message area in the lower right of the display.

$P F D$. The primary flight display contained basic airspeed and altitude tapes and attitude direction indicator (ADI). The pilot used this display to maintain the assigned flight parameters. The ADI area was used a display time-critical alerts which contained both the source of the alert (e.g., terrain) and the appropriate response to resolve the situation (e.g., arrow pointing upward).

$3 D$ audio. The aural display was used to indicate the identity and priority of the alert. Time-critical alerts were continuous, voice commands indicating the source of the threat (e.g., terrain, terrain) and the resolution (e.g., pull up, pull up). They emanated from the spatial location of the threat. Tactical alerts were also continuous alerts having unique characteristics that indicated the category of the alert and emanated from the direction of the MAESA display. Both time-critical and tactical alerts could be silenced by pressing the Master-Caution button. Strategic alerts maintained the same aural characteristics of the tactical alert but were nonrepeating and emanated from the outboard side of the pilotnot-flying.

\section{RESULTS AND DISCUSSION}

Several performance measures were collected to assess pilot performance in the different display conditions. These measures included: deviations in heading, altitude and speed from the assigned the flight path, recognition of the type of alert, detection response time and recognition accuracy to indicated threats, situation awareness measures, and preference measures. Another data point was the closest distance the pilot ever came to the hazard. Measures were used to make comparisons between concepts, with statistical differences or consistent trends indicative of relative advantages or disadvantages. For the sake of brevity, only a subset of these data is reported. For more information, see Ververs, Dorneich, Good, Rye, Downs, Neihus, and Dewing, 2001.

The flight path tracking data included: Root Mean Square Error (RMSE) data for airspeed, lateral deviation, and vertical deviation. The maximum deviation from the assigned flight path for airspeed, lateral and vertical away from the assigned path was also calculated.

There were pilot performance data points we collected in order to analyze how well they were performing on their tasks when interfacing with the various display conditions, which include:

- $\quad$ Master Reaction Time (RT) - Response time to pressing the Master Caution-Warning $(\mathrm{C}-\mathrm{W})$ Annunciator light after receiving an alert

- Recognition RT - Response time to identifying the Category of the incoming alert

- Resolution RT - Time to resolve the conflict

- Number of button presses - Total number of button presses for each scenario

Significant main effects of integration for Master RT $(\mathrm{F}(1,137)=4.89, \mathrm{p}=0.05)$ and number of button presses $(\mathrm{F}(1,106)=5.80, \mathrm{p}<0.05$ were found. Pilots were slower at initially silencing the Master Caution-Warning in the integrated vs. the separate condition $(7.78 \mathrm{sec}$ vs. $6.49 \mathrm{sec})$. It is reasoned that the pilot's visual attention was initially drawn to the integrated display to gather the information rather than the Master Caution-Warning display. In the separate condition, pilots were required to immediately interact with the display by pressing the function keys to display the appropriate information and mentally integrate the information. The Master C-W buttons were collocated to the function keys and led to the faster RTs. The number of buttons pressed is also consistent with this explanation. Pilots made more button presses in the separate display condition than the integrated condition (17.6 vs. 12.2). No consistent or interpretable main effects or interactions were found for the other measures or for the category condition.

Some additional noteworthy results involved a significant main effect of scenario for each of the above pilot performance variables. The criticality of the situation (e.g., strategic, tactical, or time-critical) and the amount of data presented (single events vs. multiple events) influenced how the pilots responded to the situation. In the scenarios where multiple sources of information needed to be integrated pilots were required to make a large number of button presses. This was particularly true in the separate display condition. Pilots had to work harder to gather the information when the displays were separated as compared to the integrated display. Pilots also perceived their workload to be significantly higher in the separate display conditions than in the integrated conditions leading to higher workload scores (4.9 vs. 3.9), 
$\mathrm{F}(1,101)=5.81, \mathrm{p}<0.05$. The added effort of pressing buttons increased the pilots' level of frustration with the displays. Again, this was particularly evident for the non-integrated condition. One pilot remarked, "I agree that it is informative to have the [advance weather] information if it doesn't take a lot of button pushing to get it, which in this scenario [Scenario 7], it did! [emphasis in pilot's original notes] I do not like the separated displays!"

Scenario 7, in particular, emphasized the main differences found in the Integration variable (integrated vs. separate display). It involved a convective weather storm cell that was developing near the destination airport. The pilot was alerted to the storm several hundred miles away. Winds reveal that the storm would progress toward the destination airport. The data revealed greater lateral and vertical deviations in the separate display condition. See Figures 2 and 3. There were many more button presses in the separate display condition and higher levels of workload as compared to the integrated display condition leading to greater pilot frustration. The pilots felt that the information about the potential weather hazards at their destination was informative, but they felt that navigating the MAESA display to look ahead in the flight plan was much easier and more intuitive in the integrated condition as opposed to the separate condition.

\section{Scenario 7: Lateral Deviation}

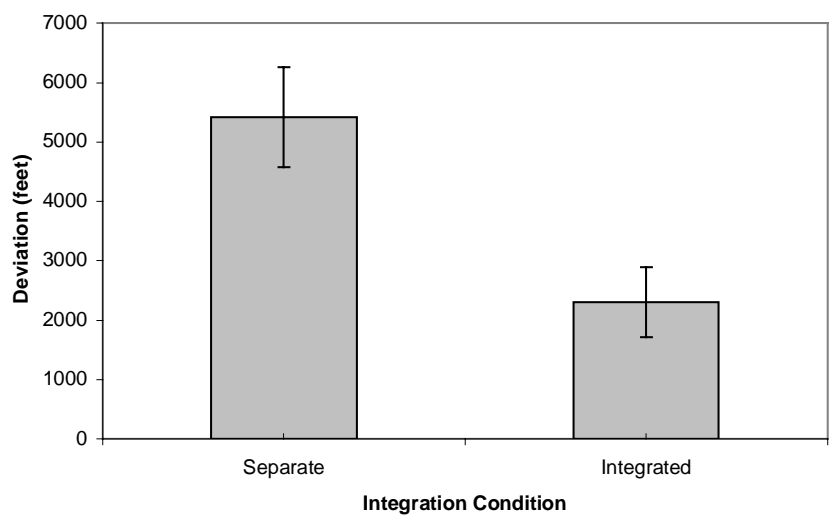

Figure 2. Lateral deviation from course in Scenario 7

Scenario 7: Vertical Deviation

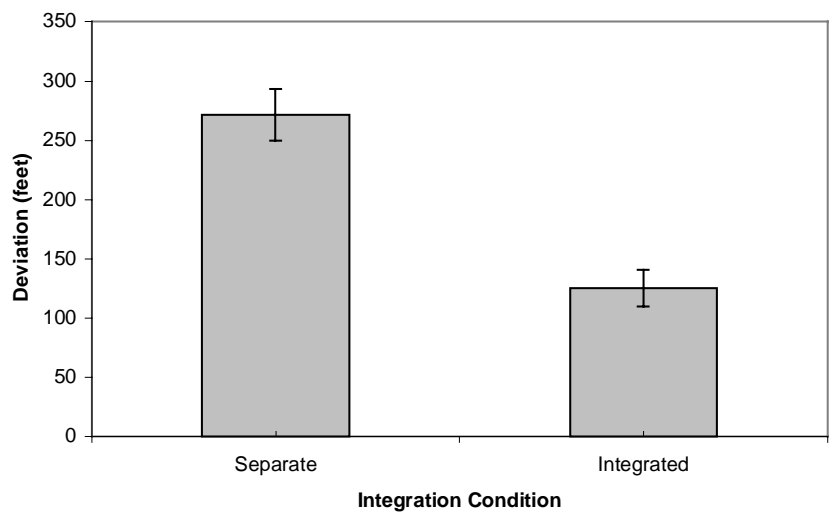

Figure 3. Vertical deviation from course in Scenario 7
A variety of scenarios were used from strategic messages advising of a gate change at the destination to complex situations involving multiple, time-critical alerts occurring simultaneously. The variation in scenario resulted in differences in all the performance measures from the pilot response to the initial alert to the workload rating scores. Below we highlight some of the results from the scenarios.

For the scenarios with time-critical alerts, the pilots' response time to turning off the audio was delayed. Clearly, the additional information provided in the voice message was advantageous for the pilot to respond to the alert. Pilots may have been too preoccupied to bother to turn off the aural annunciation. In these same scenarios, pilots were faster at resolving the alert than in tactical or strategic alert situations. There is simply more time in the latter scenarios for the pilots to make decisions. Perceived workload also varied by scenario. Strategic advisory scenarios (i.e., gate change) resulted in lower workload scores, single alert condition scenarios yielded slightly higher scores, and the multiple alert situations caused the highest scores.

Scenarios also affected the flight path tracking results (airspeed RMSE, vertical RMSE, and lateral RMSE, maximum deviation from airspeed, altitude, and heading targets). Higher priority alert scenarios caused greater deviations from the flight path than the strategic advisory scenarios. This was particularly true for airspeed. With the advanced warning in scenarios where the alerts were strategic, the pilots were dealing with future hazards and avoided a large deviation away from their assigned flight path. The maximum deviation variables revealed that the largest deviations came from the lateral maneuvers, even in situations where a much smaller vertical deviation would have cleared the alert. This provides some evidence to support the development of a vertical profile display (VPD), since vertical maneuvers would have resulted in less of a deviation off course.

Though many scenarios revealed an effect of integration, some scenarios did not. If a single alert was issued and information did not need to be integrated, pilots performed equally well in both display conditions. However, when information needed to be analyzed for their relationships across hazard types (terrain and traffic or weather and map information), pilots greatly preferred the integrated display.

\section{CONCLUSIONS}

One overarching finding of this study was the strong and consistent effect of scenario. All of the data clearly indicated that the variation on scenario was an effective manipulation to generate a variety of responses from the pilots. Many pilots commented that the scenarios were realistic and adequately represented in Honeywell Labs' simulator. It was found that those scenarios with the higher priority, time-critical alerts lead to higher workload scores. Pilots agreed that the added information in the voice alert identification and response was effective and therefore used for a longer period of time before can silencing the alert than the lower priority tactical alerts. Pilots found the use of the PFD for the presentation of timecritical information useful since that was where their attention was primarily focused. An additional finding was that strategic scenarios, where greater than 10 minutes existed before the 
threat was encountered, lead to smaller departures from their assigned course. The added time and preview of the situation proved effective and was a contributing factor in minimizing the flight path deviations. Pilots agreed that the availability of the advance information concerning the developing situation was an informative feature of the concept.

One of the main purposes of the experimental evaluation was to assess the effectiveness of information integration within a new conceptual framework and the implementation of that concept. Though the integrated condition did not show evidence of improving response times to alerts, it did lead to fewer physical interactions with the display, lower workload scores and lower frustration levels as compared to the separate display condition. Testing needs to be continued to further assess the performance advantages of alerting integration. Current research needs include the assessment of the complex prioritization of multiple conflicting alerts, the extensibility of the ANCOA concept to the development of new technologies and the feasibility of fully integrating this concept into a current or future flight deck.

\section{ACKNOWLEDGMENTS}

This work was funded by a NASA contract NAS100107, for which Drs. Christine and Celeste Belcastro served as technical monitors. It was also partially funded by Dr. Tina Beard of NASA Ames Research Center through the Aviation Safety Program's System Wide Accident Prevention program. The authors wish to thank Jeff Rye for his invaluable assistance in programming the simulation and data processing $\log$ files.

\section{REFERENCES}

Hart, S. G. and Staveland, L. E. (1988). Development of a multidimensional workload rating scale: Results of empirical and theoretical research. In P. Hancock \& N. Meshkati (Eds.), Human Mental Workload, 139-183. Amsterdam, The Netherlands: Elsevier.

Lee, J.D. and Moray, N. (1992). Trust, Control Strategies and Allocation of Functions in Human-machine Systems. Ergonomics, 35(10), pp. 1243-1270.

Parasuraman, R., and Riley, V. (1997). Humans and Automation: Use, Misuse, Disuse, Abuse. Human Factors, 39(2), pp. 230253.

Pritchett, A. R., Vandor, B., and Edwards, K. (1999). Testing and Implementing Cockpit Alerting Systems, Presented at the International Workshop on Human Error, Safety, and System Design. Liege, Belgium, June 1999. Selected for publication in Reliability Engineering and System Safety.

Riley, V. (1996). Operator reliance on Automation: theory and Data. In Automation and Human Performance: Theory and Applications, R. Parasuraman, \& M. Mouloua, (Eds.). Mahwah, NJ: Lawrence Erlbaum, pp. 19-36.

Ververs, P. M., Dorneich, M. C., Good, M. D., Rye, J., Downs, J., Niehus, G., Dewing, W. (2001, November). Alerting and Notification of Conditions Outside the Aircraft (ANCOA): Final Report. NASA Contract No. NAS1-00107. Hampton, VA: NASA Langley Research Center.

Ververs, P. M., Good, M. D., Rogers, W. H., Riley, V., and Dorneich, M. C. (September, 1999). Alerting and notification of conditions outside the aircraft: Concept defined and prototyped. NASA Contract No. NAS1-20219. Hampton, VA: NASA Langley Research Center.

Woods, D.D. (1986). Paradigms for intelligent decision support. Intelligent Decision Support in Process Environments, NATA ASI Series, Vol. F21.

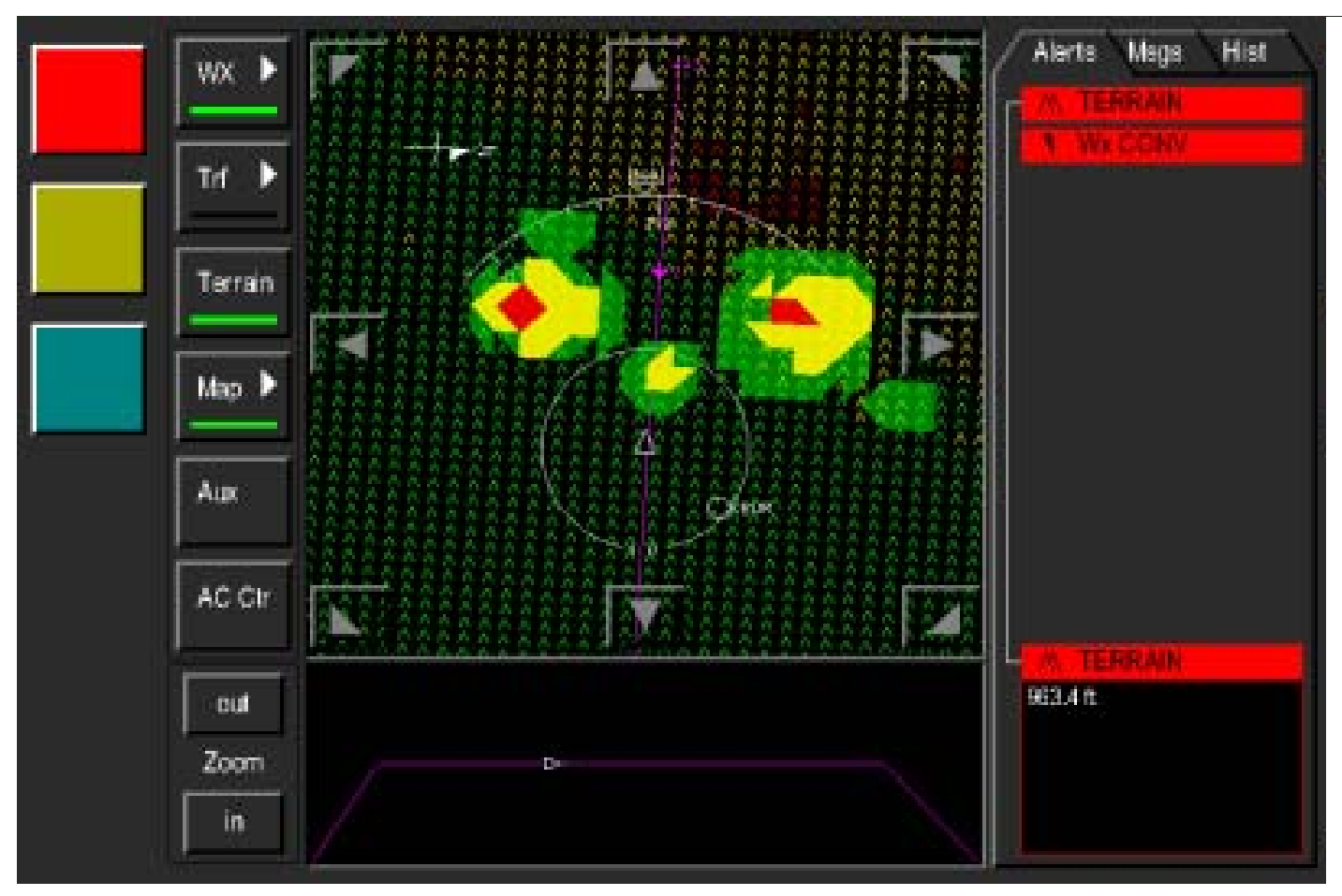

Figure 1. MAESA display depicting weather and terrain information in the integrated condition 